\title{
Etude d'un lot de ferments lactiques mésophiles. Equilibre des flores au cours de la première étape de la fabrication du levain
}

\author{
S. Lucas et J. Reyrolle
}

IRBA, Institut de recherche en biologie appliquée, Equipe de génétique microbienne, université de Caen, 14032 Caen Cedex, France

(reçu le 17-3-1988, accepté le 19-10-1988)

Résumé - Grâce à une méthodologie simple : choix de quelques caractères d'identification et utilisation d'un ensemenceur multipoint, nous avons, d'une part contrôlé la flore d'un lot de ferments lactiques mésophiles, d'autre part vérifié la reproductibilité du premier repiquage de la fabrication d'un levain, telle qu'elle est pratiquée dans l'industrie laitière. Nous avons également fait varier la température d'incubation $\left(24\right.$ ou $28^{\circ} \mathrm{C}$ au lieu de $26^{\circ} \mathrm{C}$ ) et nous avons arrêté les cultures à des acidités plus fortes $\left(75^{\circ} \mathrm{D}\right.$ au lieu de $\left.65^{\circ} \mathrm{D}\right)$.

Nous constatons qu'il n'y a pas de variations quantitatives ou qualitatives des flores à l'intérieur du même lot de ferments. La première étape est reproductible en ce qui concerne les différentes espèces détectées si les conditions d'incubation sont identiques. En revanche, des variations de température et/ou d'acidité finale entraînent des modifications importantes des flores, spécialement pour les espèces : Streptococcus lactis ssp. diacetylactis et Streptococcus cremoris.

ferment - levain - Streptococcus lactis - Streptococcus cremoris - Streptococcus lactis ssp. diacetylactis - acidification - température d'incubation

Summary - Study of a mesophilic lactic acid bacteria starter batch. Evolution of flora balance during the first stage of starter propagation. A simple methodology : the utilization of a multipoint inoculator and the selection of some characteristics of identification have permitted to control a mesophilic lactic acid bacteria starter batch. The reproducibility of the first step of starter propagation has been verified. In a second way, we changed the incubation temperature of this first stage and the culture were stopped for another final acidity : $75^{\circ}$ Dornic instead of $65^{\circ} \mathrm{D}$ (the acidity is measured by neutralization of $10 \mathrm{ml}$ of milk, which contains phenolphtalein, by NaOH N/9: $10^{\circ} \mathrm{Dor}$ nic $=1 \mathrm{~g} / \mathrm{ll}$ lactic acid $)$.

We note that the flora are constant in the same batch of starter. Only the three species of mesophilic lactic acid streptococci are identified, Streptococcus lactis and Streptococcus cremoris represent $77 \%$ of the population. The first stage can be reproduced. The fiora are increased 72 -fold in comparison with the inoculum. The proportions of each species were modified: Streptococcus diacetylactis became the most important flora $(55.6 \%)$. When the incubation temperature and the final acidity change, we note that the equilibrium between the different species are modified. The flora of Streptococcus lactis ssp. diacetylactis and Streptococcus cremoris are subjected to variations : the 
population of Streptococcus cremoris is 4.8 fold more important to an incubation temperature of $26^{\circ} \mathrm{C}$ in comparison with $28^{\circ} \mathrm{C}$ and the final population of Streptococcus diacetylactis is higher to $65^{\circ}$ Dornic than $75^{\circ}$ Dornic.

starter - Streptococcus lactis - Streptococcus cremoris - Streptococcus lactis ssp. diacetylactis - acidification - incubation - temperature

\section{Introduction}

Les bactéries lactiques, largement étudiées de par le monde, sont utilisées dans l'industrie laitière sous différentes formes et dans des conditions très variées. Nous savons aujourd'hui que le choix d'un ferment et le judicieux équilibre des souches qui le composent sont responsables pour une bonne part de la qualité et de la régularité du produit fini.

Si certains auteurs (Cogan, 1980; Stadhouders, 1986) énumèrent les différents facteurs intervenant sur l'activité d'un levain, principalement la qualité du lait, les phages et le choix du ferment, l'évolution même des différentes espèces composant le levain lors de sa fabrication est plus rarement évoquée. II semble que l'intérêt se focalise autour des phénomènes technologiques comme l'acidification, les attaques phagiques (Leach et Sandine, 1975; Anonyme, 1979; Shaw, 1983), ainsi que sur les modes de propagation des levains (Huggins, 1984; Roy et al., 1987). En fait, peu d'études ont été effectuées sur les ferments mêmes, et celles qui ont été réalisées sont limitées pour des raisons expérimentales à un couple de bactéries et à une description qualitative. C'est le cas de Martley (1975) qui suit le comportement d'une ou 2 souches dans une fabrication de camembert et d'Hugenholtz et Veldkamp (1985) qui décrivent l'évolution des équilibres lors de cultures mixtes de $S$. cremoris.

Nous nous sommes donc attachés tout au long de ce travail à l'analyse qualitati- ve et quantitative des populations d'un ferment multiple mésophile, dans le but de montrer, dans un premier temps, quelles étaient exactement les espèces présentes, et quelles en étaient les proportions afin de vérifier la régularité des équilibres des flores, dans un lot de ferment commercial de ce type. Dans un second temps, nous avons cherché à connaître les variations possibles de ces équilibres lors de la première étape de la fabrication d'un levain dans des conditions proches de celles de l'industrie laitière et finalement, nous avons testé l'influence des paramètres essentiels que sont la température d'incubation et l'acidité finale avant l'arrêt de l'incubation sur ces équilibres de la flore lactique.

\section{Matériels et Méthodes}

\section{Souches}

Ce travail est réalisé sur un ferment lactique mésophile multiple du commerce se présentant sous forme lyophilisée (fournisseur Wiesby, ferment référencé : Probat 404).

\section{Milieux}

Les bases gélosées, dépourvues de sucre des milieux M17 (Terzaghi et Sandine, 1975), MRS (De Man et al., 1960), sont stérilisées par autoclavage à $120^{\circ} \mathrm{C}, 15 \mathrm{~min}$. Les solutions de sucres sont stérilisées par filtration (filtre Sartorius, type : Flow-pore $0,45 \mu \mathrm{m} ø$ ), puis ajoutées à raison d'une concentration finale de $0,5 \% \mathrm{p} / \mathrm{v}$ 
au milieu de base pour les milieux de dénombrements, et à raison de $1 \% \mathrm{p} / \mathrm{v}$ pour les milieux d'identification.

Tryptone-sel : milieu de dilution, composition : tryptone (Merck) $1 \mathrm{~g}, \mathrm{NaCl}$ (Merck) $8 \mathrm{~g}$, $\mathrm{H}_{2} \mathrm{O}$ qsp $1 \mathrm{I}$, autoclavage à $120^{\circ} \mathrm{C}, 15 \mathrm{~min}$.

\section{Dénombrement}

Après remise en suspension 10 min dans $10 \mathrm{ml}$ de milieu tryptone-sel, les échantillons sont dilués dans ce même milieu, et pour chaque dilution retenue, 3 boîtes de chacun des milieux suivants (M17 glucose, MRS lactose, MRS lactose additionné d'azoture de sodium $100 \mu \mathrm{g} / \mathrm{ml}$ ) sont ensemencées en surface puis incubées à $30^{\circ} \mathrm{C}$ en aérobiose pendant $48 \mathrm{~h}$.

La recherche systématique des lactobacilles est réalisée sur MRS glucose $0,5 \%(p / v)$, $\mathrm{pH} 5,5$ à $37^{\circ} \mathrm{C}$ en anaérobiose, ainsi que la recherche des streptocoques lactiques thermophiles et de streptocoques thermophiles autres que lactiques respectivement sur M17 lactose $0,5 \%(p / v)$ à $42{ }^{\circ} \mathrm{C}$ et sur M17 sans sucre à $42^{\circ} \mathrm{C}$.

\section{Identification}

Pour chaque milieu, après dénombrement, un nombre de colonies correspondant à la racine carrée du nombre moyen des colonies numérées (Guiraud et Galzy, 1980) est prélevé sur une des 3 boîtes (la plus proche de la moyenne) et isolé sur les mêmes milieux, dans les mêmes conditions par la méthode des stries. Après incubation, un clone de chaque isolement est identifié. Ce travail nécessitant l'identification d'un grand nombre de bactéries, nous avons choisi une méthode rapide : l'étude de la croissance sur sucres en milieu gélosé.

L'ensemencement est réalisé grâce à un ensemenceur automatique (Inoculator multipoint Denley) qui permet un travail simultané sur 25 souches, le contrôle de l'inoculum et l'ensemencement identique d'un grand nombre de boîtes (Fig. 1).

Les streptocoques lactiques sont identifiés grâce aux milieux suivants : M17 lactose, M17 glucose, M17 ribose, M17 maltose, M17 fructose; de plus, nous utilisons la capacité à produire du diacétyle sur lait pour différencier $S$. lactis ssp. diacetylactis de $S$. lactis et le test de Turner (Turner et al., 1963) adapté en milieu liquide pour mettre en évidence la présence d'une arginine désaminase chez $S$. lactis et $S$. lactis ssp. diacetylactis.

Les leuconostocs sont identifiés grâce aux milieux suivants (Garvie, 1960) : MRS lactose, MRS glucose, MRS arabinose, MRS tréhalose, MRS saccharose ainsi que par la capacité pour certaines espèces à produire des dextranes sur MRS glucose additionné de saccharose (10\% p/v).

\section{Contrôle d'un lot de ferment}

De 0,100 à $0,150 \mathrm{~g}$ de ferment lyophilisé sont prélevés dans 5 sachets issus d'un même lot. Ils sont remis en suspension pendant $10 \mathrm{~min}$ dans $10 \mathrm{ml}$ de milieu tryptone-sel puis agités au vortex pendant $4 \mathrm{~min}$ avant numération.

\section{Le lait}

C'est un lait écrémé (poudre Elle et Vire) reconstitué à raison de $100 \mathrm{~g} / \mathrm{l}$ puis chauffé à vapeurs fluentes pendant $60 \mathrm{~min}$.

\section{Protocole expérimental du premier repi- quage}

Sept flacons de $250 \mathrm{ml}$ contenant respectivement $100 \mathrm{ml}$ de lait écrémé reconstitué sont ensemencés à raison de $0,5 \% \mathrm{p} / \mathrm{v}$ à partir d'un même sachet de ferment. Trois d'entre eux sont destinés au contrôle de l'acidification (des mesures sont effectuées toutes les $2 \mathrm{~h}$ pendant les 6 premières heures puis environ toutes les demi-heures jusqu'à l'obtention de l'acidité souhaitée), les 4 autres servent à l'expérience proprement dite. L'incubation au bain-marie est réalisée à $26 \pm 0,3^{\circ} \mathrm{C}$ et poursuivie jusqu'à l'obtention d'une acidité Dornic de $65^{\circ}$. L'acidification est alors bloquée en plaçant les flacons dans de l'eau glacée à $+4^{\circ} \mathrm{C}$ jusqu'au moment de l'analyse (le lendemain), soit entre 22 et $24 \mathrm{~h}$ après l'ensemencement. Pendant cette conservation à $4{ }^{\circ} \mathrm{C}$, aucune variation de l'acidité n'a été notée.

\section{Etude de l'influence des paramètres technologiques : température, acidité}

Du lait reconstitué ensemencé à $0,5 \% \mathrm{p} / \mathrm{v}$ à partir d'un même sachet est réparti dans 2 séries de 18 tubes, à raison de $10 \mathrm{ml}$ par tube. Une série de tubes est incubée au bain-marie à 


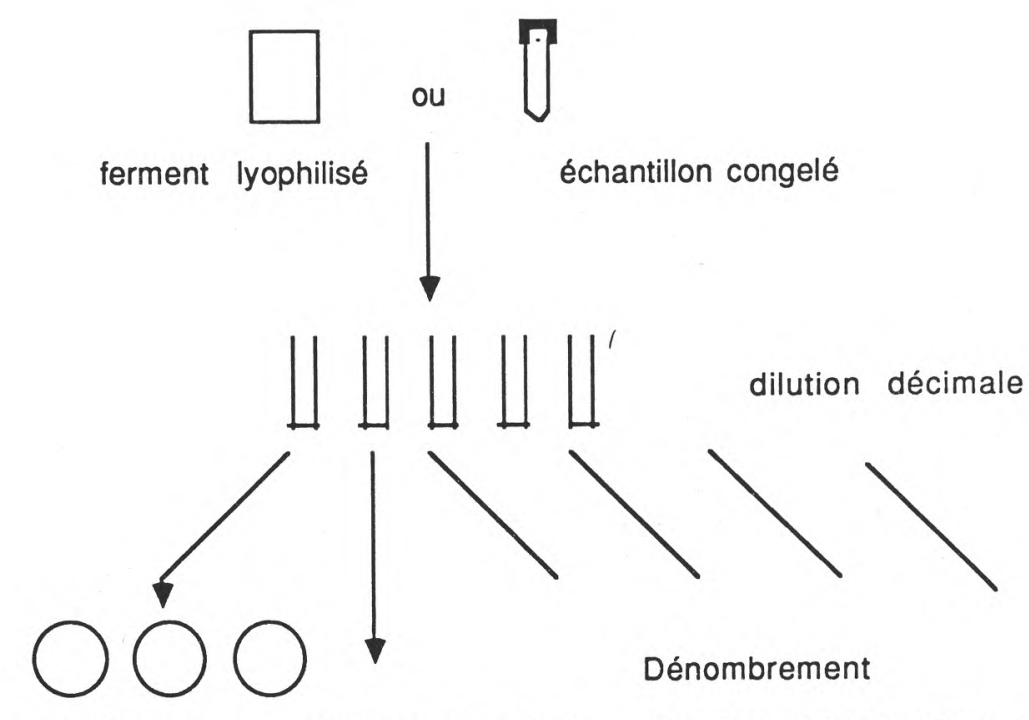

M17 GLU 0,5\%

MRS LAC MRS LAC AZ

MRS GLU M17 LAC M17 Sans sucre
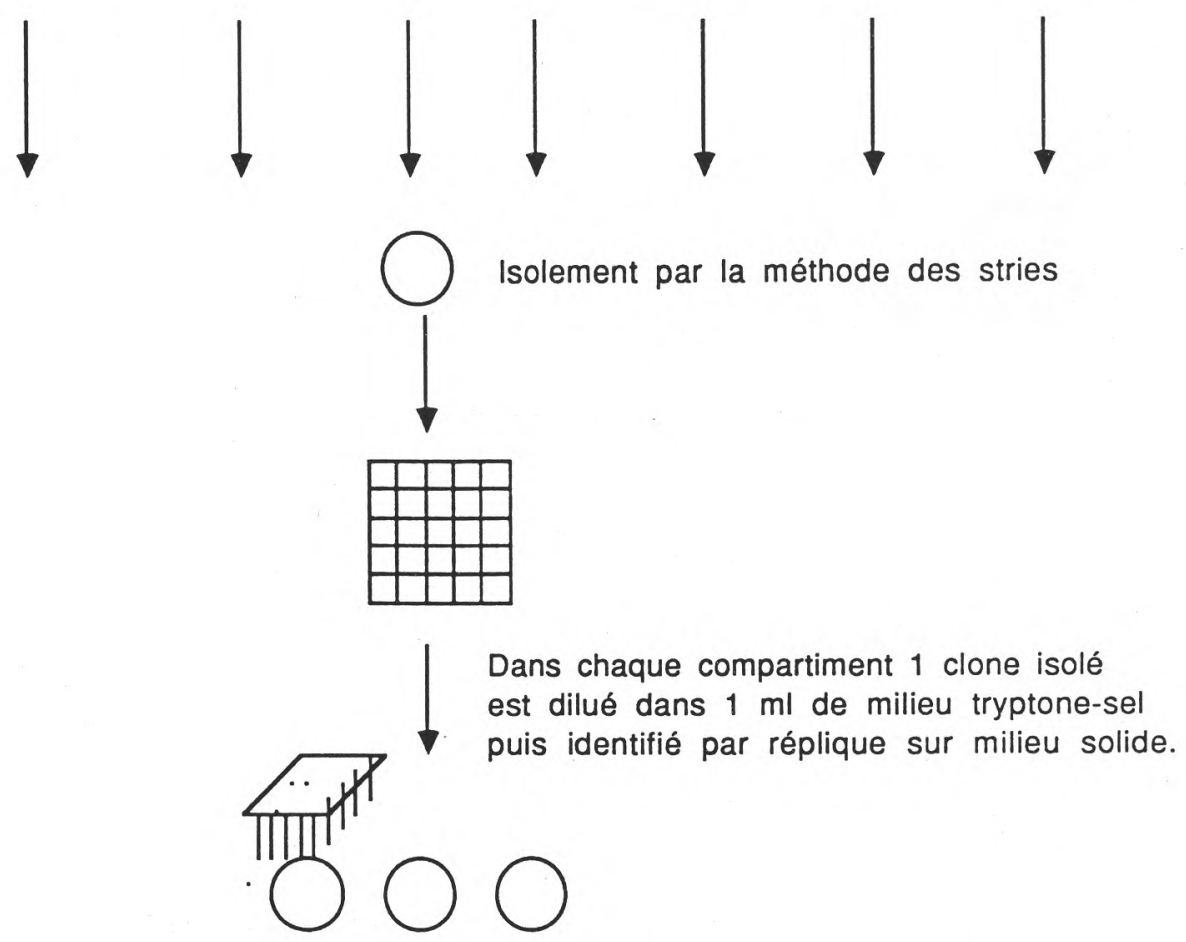

Fig. 1. Protocole de dénombrement et d'identification. 
$28 \pm 0,3^{\circ} \mathrm{C}$, une autre à $24 \pm 0,3^{\circ} \mathrm{C}$. Pour chaque série, 2 tubes sont bloqués dans de l'eau glacée $\left(+4^{\circ} \mathrm{C}\right)$ pendant une nuit avant analyse des populations dès que l'acidité atteint $65^{\circ} \mathrm{D}$; il en est de même pour 2 autres tubes de chaque série pour une acidité de $75^{\circ} \mathrm{D}$. Les 28 autres tubes servent au contrôle et au suivi de l'acidification. Les échantillons conservés une nuit sont ensuite analysés dans un laps de temps inférieur à $2 \mathrm{~h}$ (entre 22 et $24 \mathrm{~h}$ après l'ensemencement).

\section{Mesure de l'acidification}

Elle est suivie soit par mesure de l'acidité Dornic, soit par $\mathrm{pH}$-métrie.

L'acidité Dornic. C'est une expression de l'acidité développée dans un lait par transformation du lactose en acide lactique. La mesure de l'acidité Dornic est réalisée par neutralisation, par de la soude $\mathrm{N} / 9$, de $10 \mathrm{ml}$ de lait auquel est ajouté un indicateur de $\mathrm{pH}$ (la phénolphtaléine); $10^{\circ} \mathrm{D}=1 \mathrm{~g} / \mathrm{d}$ d'acide lactique.

\section{Résultats}

\section{Contrôle d'un lot de ferment}

Lors de cette première étude, nous avons cherché à vérifier que les sachets de fer- ments issus d'un même lot avaient une composition similaire. Cela afin de s'assurer que le stockage de ces ferments n'intervient pas significativement sur les équilibres bactériens, en vue d'éliminer lors de nos expériences les éventuelles interférences provoquées par des variations de population dans un même lot. Les résultats concernant 5 sachets testés issus d'un même lot sont consignés dans le Tableau I, les valeurs et moyennes sont exprimées en logarithmes décimaux compte tenu du type d'échantillons analysés et donc des variances importantes qu'ils entraînent.

Au cours du travail d'identification, il est apparu que toutes les souches n'avaient pas les caractéristiques typiques des différentes espèces de streptocoques lactiques mésophiles (Deibel et Seiley, 1974; Jones, 1978; Garvie et Farrow, 1982). II existe ce que nous pourrions appeler des variants biochimiques qui ne diffèrent des souches-types que par un, voire deux, caractères. Nous avons donc retenu les critères biochimiques d'identification résumés dans le Tableau II.

Les flores totales (nombre de bactéries/g ferment) sont identiques, la valeur

Tableau I. Répartition des espèces dans un ferment multiple (expérience réalisée pour 5 sachets d'un même lot).
S. diacetylactis
S. lactis
S. cremoris
Flore totale

\begin{tabular}{lllll} 
Lot $A^{\otimes}$ & 8,84 & 8,30 & 8,90 & 9,23 \\
Lot $B^{\otimes}$ & 8,95 & 8,88 & 8,92 & 9,40 \\
Lot $C^{\otimes}$ & 8,72 & 8,42 & 9,04 & 9,28 \\
Lot $^{\otimes}$ & 9,03 & 8,70 & 9,11 & 9,46 \\
Lot $^{\otimes}$ & 8,92 & 8,18 & 9,08 & 9,34 \\
Moyenne & $8,90 \pm 0,10$ & $8,50 \pm 0,26$ & $9 \quad \pm 0,08$ & $9,34 \pm 0,08$ \\
Population réelle & $7,8.10^{8}$ & $3,1.10^{8}$ & $10,3.10^{8}$ & $21,1.10^{8}$ \\
$\%$ population totale & 36,8 & 14,6 & 48,6 & 100 \\
\hline
\end{tabular}


moyenne étant de $2,1.10^{9}$ bactéries/g. A l'exception des streptocoques lactiques mésophiles, aucune autre flore recherchée n'a été détectée : streptocoques thermophiles, streptocoques autres que lactiques, lactobacilles et leuconostocs (- de 10 bactéries/g). En ce qui concerne les streptocoques lactiques mésophiles, la répartition des espèces identifiées selon les critères précédents montre une flore acidifiante majoritaire $(63 \%$ environ de la flore totale dont $77 \%$ de S. cremoris, et une flore aromatique composée de $S$. lactis ssp. diacetylactis. L'importance de la population de chaque espèce est identique, quel que soit le sachet considéré.

Evolution des populations au cours $d u$ premier repiquage

Les résultats concernant les populations dénombrées à l'issue de la période d'incubation de ce premier repiquage sont consignés dans le Tableau III. Les critères d'identification restent les mêmes que ceux utilisés dans la première partie de ce travail. Les flores totales des 4 essais, obtenus à partir d'inoculums provenant de 4 échantillons différents, ne diffèrent pas significativement. La valeur moyenne de cette population est de $7,5.10^{8}$ bactéries/ml, soit une augmentation de 72 fois par rapport à l'inoculum (Tableau IV).

Cette homogénéité se retrouve lorsque l'on examine les résultats concernant les populations finales atteintes par chaque espèce pour les 4 fermentations (Tableau III). En revanche, si nous comparons les proportions relatives de ces espèces entre le début (pourcentage de chaque espèce dans l'inoculum) et la fin des fermentations, nous observons des variations dans les proportions respectives des différentes espèces. En particulier, la population de $S$. lactis ssp. diacetylactis qui représente $36,8 \%$ de la population totale de l'inoculum devient majoritaire, avec un pourcentage de $55,6 \%$ (Tableau IV).

Influence des paramètres : température, acidité

\section{Influence de la température d'incubation}

Dans cette expérience, nous comparons l'effet de la température d'incubation sur l'évolution des flores pour une même acidité finale de $65^{\circ} \mathrm{D}$. Les concentrations finales en cellules viables des populations

Tableau II. Critères d'identification des streptocoques lactiques mésophiles.

$\begin{array}{llll}\text { Croissance sur } & \text { S. lactis ssp. diacetylactis } & \text { S. lactis } & \text { S. cremoris }\end{array}$

Lactose
Glucose
Ribose
Maltose
Fructose
Tréhalose
Hydrolyse de l'arginine
Utilisation du citrate

$\begin{array}{lll}+ & + & + \\ + & + & + \\ + & + & - \\ + & + & \pm \\ + & + & + \\ \pm & \pm & \pm \\ + & + & - \\ + & - & +\end{array}$

+ : utilisation du sucre ou présence d'une arginine désaminase; - : pas d'utilisation du sucre ou absence d'une arginine désaminase; \pm : utilisation variable selon les souches. 
Tableau III. Répartition des espèces après un premier repiquage du ferment (4 essais indépendants).
S. diacetylactis
S. lactis
S. cremoris
Flore totale

\begin{tabular}{lllll} 
Essai $n^{\circ} 1^{\otimes}$ & 8,69 & 7,85 & 8,40 & 8,91 \\
${\text { Essai } n^{\circ} 2^{\otimes}}^{\text {Essai } n^{\circ} 3^{\otimes}}$ & 8,56 & 7,90 & 8,45 & 8,85 \\
${\text { Essai } n^{\circ} 4^{\otimes}}^{\text {Moyenne }}{ }^{\otimes}$ & 8,59 & 7,90 & 8,28 & 8,82 \\
& 8,64 & 8,23 & 8,40 & 8,93 \\
Population réelle* & $8,62 \pm 0,05$ & $7,97 \pm 0,15$ & $8,38 \pm 0,06$ & $8,88 \pm 0,04$ \\
\% population totale & $4,17.10^{8}$ & $0,93.10^{8}$ & $2,40.10^{8}$ & $7,54.10^{8}$ \\
\hline
\end{tabular}

${ }^{\star}$ : moyenne exprimée en bactéries par ml. ${ }^{\otimes}$ Log (population bactéries/ml).

obtenues à 24 et $26^{\circ} \mathrm{C}$ sont semblables, une légère baisse est enregistrée à $28^{\circ} \mathrm{C}$ (Tableau V).

Cette homogénéité relative des flores totales masque des variations des populations de 2 espèces : il y a 1,7 fois plus de $S$. lactis après incubation à $28^{\circ} \mathrm{C}$ comparativement à $26^{\circ} \mathrm{C}$, et 4,8 fois plus de $S$. cremoris à $26{ }^{\circ} \mathrm{C}$ par rapport à une température de $28^{\circ} \mathrm{C}$. Ces variations sont à corréler avec la diminution de la flore globale à $28^{\circ} \mathrm{C}$. Cela peut s'expliquer en fonction des courbes d'acidification (Fig. 2), par le fait que les cellules cultivées à $28^{\circ} \mathrm{C}$ atteignent plus rapidement $65^{\circ} \mathrm{D}$, et séjournent donc plus longtemps dans cet environnement acide, entraînant une diminution de leur viabilité. Les souches de $S$. cremoris sont les plus sévèrement touchées. Une autre hypothèse serait que les taux de croissance des souches de chacune des 3 espèces dans le lait sont suffisamment différents selon la température d'incubation (Lee et Collins, 1976) pour entraîner de telles variations.

\section{Influence de l'acidité finale}

Le fait de prolonger l'incubation de 65 à $75^{\circ} \mathrm{D}$ implique 2 remarques (Tableau VI).

Tableau IV. Comparaison des flores de l'inoculum et de celles de fin du premier repiquage.

$\begin{array}{lllll}\begin{array}{l}\text { Population } \\ \text { de l'inoculum * }\end{array} & \% & \text { Population } & \% & \text { Nbre } \\ \text { flore totale } & \text { en fin d'incubation * } & \text { flore totale de générations }\end{array}$

$\begin{array}{llll}\text { Population totale } 1,05.10^{7} & 100 & 7,54.10^{8} & 100\end{array}$

\begin{tabular}{llllll} 
S. lactis & $1,53.10^{6}$ & 14,6 & $0,93.10^{8}$ & 12,4 & 5,9 \\
S. lactis ssp. & & & & & \\
\multicolumn{1}{c}{ diacetylactis } & $3,86.10^{6}$ & 36,8 & $4,17.10^{8}$ & 55,6 & 6,7 \\
S. cremoris & $5,10.10^{6}$ & 48,6 & $2,39.10^{8}$ & 32 & 5,5
\end{tabular}


Tableau V. Influence de la température d'incubation sur les équilibres bactériens.

\begin{tabular}{lllll}
\hline & & \multicolumn{2}{l}{$\begin{array}{l}\text { Température d'incubation } \\
\text { du ferment }\end{array}$} & \\
& & $20^{\circ} \mathrm{C}$ & $26^{\circ} \mathrm{C}$ & $2{ }^{\circ} \mathrm{C}$ \\
\hline & & & & \\
$\begin{array}{c}\text { Population de } \\
\text { chaque espèce } \\
\text { détectée }\end{array}$ & S. lactis ssp. diacetylactis* & 8,59 & 8,62 & 8,56 \\
& S. lactis* & 8,57 & 7,97 & 8,21 \\
& S. cremoris* & 8,12 & 8,38 & 7,70 \\
& Population totale * & 8,89 & 8,88 & 8,74 \\
\hline
\end{tabular}

*: Log (bactéries/ml).

D'une part, nous constatons une légère diminution de la flore totale quelle que soit la température d'incubation et d'autre part, cette incubation prolongée a des conséquences différentes selon l'espèce considérée : les souches de $S$. lactis semblent peu atteintes, alors que $S$. lactis ssp. diacetylactis et surtout $S$. cremoris sont sensibles à ce maintien à des acidités élevées, et leur viabilité diminue, de façon drastique pour S. cremoris.

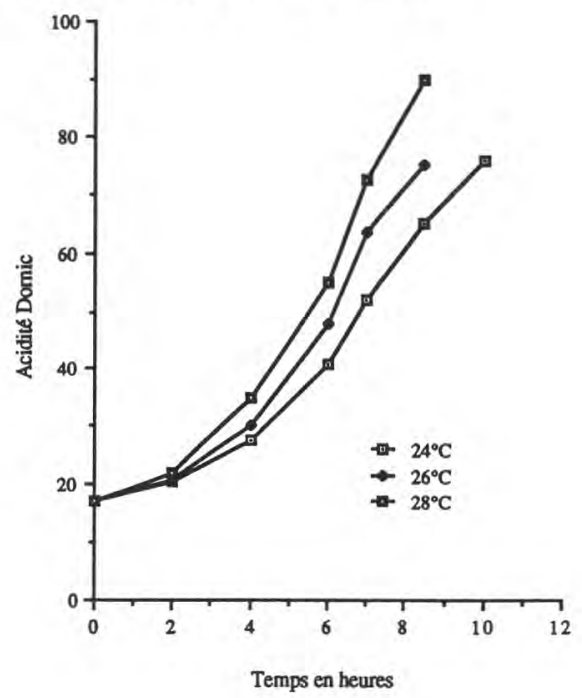

Fig. 2. Evolution de l'acidité en fonction de la température d'incubation.

\section{Discussion et Conclusion}

Nous avons, au cours de ce travail, suivi l'évolution d'un mélange complexe de souches au cours de cultures sur lait. Cela a pu être fait sur un plan quantitatif grâce à une méthodologie relativement précise et peu coûteuse. L'identification biochimique des souches, étape nécessaire tant que des techniques plus rapides ne peuvent s'appliquer qu'à certaines souches choisies (Hugenholtz et al., 1987; Boquien et al., 1988), est ici simplifiée et accélérée par l'emploi de l'inoculation multiple sur milieux gélosés renfermant différents substrats carbonés.

Cette technique, appliquée à l'étude d'un lot de ferment commercial lyophilisé de bactéries lactiques mésophiles, a permis de mettre en évidence l'homogénéité du produit proposé tant sur le plan qualitatif que quantitatif. Cette première vérification faite, nous avons reproduit au laboratoire la première étape de fabrication d'un levain en nous plaçant dans des conditions de température et d'acidité finale identiques à celles utilisées dans une unité industrielle : température d'incubation $26^{\circ} \mathrm{C}$, arrêt de la culture à $65^{\circ} \mathrm{D}$ par immersion dans l'eau glacée et analyse de la population $22 \mathrm{~h}$ après l'ensemencement. 
Tableau VI. Influence de l'acidité finale sur les équilibres bactériens.

Température d'incubation

du ferment

$24^{\circ} \mathrm{C}$

$28^{\circ} \mathrm{C}$

Acidité finale de la culture

$65^{\circ} \mathrm{D}$

$75^{\circ} \mathrm{D}$

$65^{\circ} \mathrm{D}$

$75^{\circ} \mathrm{D}$

$\begin{array}{lllll}\text { S. lactis ssp. diacetylactis* } & 8,59 & 8,39 & 8,56 & 8,34 \\ \text { S. lactis } & 8,57 & 8,47 & 8,21 & 8,21 \\ \text { S. } \text { cremoris }^{*} & 8,12 & 7,38 & 7,7 & a \\ \text { Population totale* }^{*} & 8,89 & 8,76 & 8,74 & 8,59\end{array}$

"; Log (bactéries/ml); a : population $<10^{7}$ bactéries $/ \mathrm{ml}$.

Les résultats obtenus mettent en évidence que, bien que les proportions relatives des différentes souches de $S$. lactis, $S$. lactis ssp. diacetylactis et $S$. cremoris soient différentes entre l'inoculum (ferment lyophilisé) et la culture finale, ces proportions sont remarquablement constantes en fin d'incubation entre les différents essais réalisés dans les mêmes conditions expérimentales. En revanche, une modification de la température d'incubation entraîne des variations notables dans la répartition des différentes flores et le maintien de la viabilité de ces souches en présence d'une acidité élevée (Ross, 1980). En effet, plus la température d'incubation est élevée, plus l'acidité finale fixée est atteinte rapidement, et par conséquent les souches sont exposées plus longtemps à cet environnement hostile avant leur analyse ou leur utilisation en fromagerie $22 \mathrm{~h}$ après l'inoculation. II en ressort qu'afin d'assurer un équilibre constant entre les différentes espèces d'un levain multiple, le fromager devra veiller particulièrement au respect des conditions qu'il s'est imposé à chacune des étapes de la fabrication de ce levain.
II est vraisemblable que les effets mis en évidence lors de la première étape de la fabrication d'un levain se retrouvent au cours des étapes suivantes de sa propagation, et conduisent à des modifications des flores qu'il convient de contrôler le plus possible.

\section{Remerciements}

Nous remercions le service $R$ \& $D$ de l'Union Laitière Normande qui nous a permis de réaliser ce travail et A. Birette (IUT de Caen, option $(A B)$, pour sa participation technique dans le cadre de son stage de fin d'étude.

\section{Références}

Anonyme (1979) Une table ronde de la technique laitière. Tech. Lait. 939, 13-27

Boquien C.Y., Desmazeaud M.J. \& Corrieu G. (1988) Suivi des populations mixtes de bactéries lactiques mésophiles utilisées en industrie laitière. Colloque Alimentation Transformation des Produits agricoles et Nutrition. APRIA, Paris 
Cogan T.M. (1980) Les levains lactiques mésophiles. Une revue. Lait 60, 397-425

Deibel R.H. \& Seiley H.W. (1974) Family II Streptococacceae. In : Bergey's Manual of Determinative Bacteriology. 8th ed. (Buchanan R.E. \& Gibbons N.E., eds), The Williams and Wilkins Co, Baltimore

De Man J.C., Rogosa R. \& Sharpe M.E. (1960) A medium for the cultivation of Lactobacilli. $J$. Appl. Bacteriol. 23, 130-135

Garvie E.I. (1960) The genus Leuconostoc and its nomenclature. J. Dairy Res. 27, 283-292

Garvie E.I. \& Farrow A.E. (1982) Streptococcus lactis subsp. cremoris (Orla Jensen) comb. nov. and Streptococcus lactis subsp. diacetylactis (Matuszewski et al.) nom. rev., comb. nov. Int. J. Syst. Bacteriol. 32, 453-455

Guiraud J. \& Galzy P. (1980) L'Analyse Microbiologique dans les Industries Alimentaires. Editions de l'Usine Nouvelle, p. 104

Jones D. (1978) Composition and differentiation of the genus Streptococcus. In : Streptococci (Skinner F.H. \& Quesnel L.B., eds) Academic Press, London, pp. 1-49

Hugenholtz J., Veldkamp H. \& Konings W.N. (1987) Detection of specific strains and variants of Streptococcus cremoris in mixed cultures by immunofluorescence. Appl. Environ. Microbiol. 53, 149-155

Hugenholtz J. \& Veldkamp H. (1985) Competition between different strains of Streptococcus cremoris. FEMS Microbiol. Ecol. 31, 57-62

Huggins A.R. (1984) Progress in dairy starter culture technology. Food Technol. 38, 41-45
Leach R.D. \& Sandine W.E. (1975) Numerical relationship between strains in frozen concentrates of streptococcal starter cultures. J. Dairy Sci. 59, 1392-1397

Lee D.A. \& Collins E.B. (1976) Influences of temperature on growth of Streptococcus cremoris and Streptococcus lactis. J. Dairy Sci. 59, 405-409

Martley F.G. (1975) Comportement et rôle des streptocoques lactiques du levain en fabrication de camembert. Lait 55, 310-323

Ross G.D. (1980) Observations on the effect of inoculum $\mathrm{pH}$ on the growth and acid production of lactic streptococci in milk. Aust. J. Dairy Technol. 35, 147-149

Roy R., Goulet J. \& Provencher P. (1987) Influence de 4 milieux de propagation sur le comportement de 6 levains lactiques mésophiles du commerce. Lait 67, 41-50

Shaw M. (1983) La lutte contre les bactériophages dans les fromageries. Tech. Lait. 976, 51-59

Stadhouders J. (1986) The control of cheese activity. Neth. Milk Dairy J. 40, 155-176

Terzaghi B.E. \& Sandine S.E. (1975) Improved medium for lactic streptococci and their bacteriophages. Appl. Microbiol. 29, 807-813

Turner N., Sandine W.E., Elliker P.R. \& Day E.A. (1963) Use of tetrazolium dyes in an agar medium for differentiation of Streptococcus lactis and Streptococcus cremoris. J. Dairy Sci. $46,380-385$ 